\title{
Introduction to Special Issue on "Innovating Actuarial Research on Financial Risk and Enterprise Risk Management"
}

\author{
Marcello Galeotti ${ }^{1}$
}

/ Accepted: 26 May 2021 / Published online: 3 June 2021

(c) Associazione per la Matematica Applicata alle Scienze Economiche e Sociali (AMASES) 2021

The special issue of DEF dedicated to "Innovating Actuarial Research on Financial Risk and Enterprise Risk Management” assembles 10 articles elaborating talks delivered at the AFIR/ERM (Actuarial Approach for Financial Risks and Enterprise Risk Management) Colloquium held in Florence in May 2019. In fact, these contributions, moving from both classic insurance-financial topics (longevity risks, lapse rates, Black and Scholes model, variable annuities) and far more recent actuarial fields (cyber risks, flood risk resilience, reverse mortgages), show some common features which stand out as innovative approaches and techniques. Such novelties can be classified under three typologies.

1. A dynamic and stochastic approach versus a more traditional static and deterministic one.

2. The extensive use of machine learning, artificial intelligence and other updated computational techniques.

3. The design of new types of insurance and financial contracts adequate to the above conceptual and technological innovations.

Coming to the single articles, I will try to briefly illustrate the main themes in each of them.

- An application of sigmoid and double-sigmoid functions for dynamic policyholder behavior (Baione, Biancalana, De Angelis). The authors underline the growing relevance of risk-based valuations of insurance contracts. That, in particular, causes a shift from deterministic lapse rate models to dynamic ones. In fact, most models assume a monotonic increase in lapse rate by increasing an economic variable, set equal to a "market spread" between a benchmark rate and the policy crediting rate. In the article, a different policyholder behavior is proposed, assuming the behavior is not modified for small values of the market spread. In order to better

Marcello Galeotti

marcello.galeotti@unifi.it

1 Florence, Italy 
describe the consequences of such assumption, a double-sigmoid function, versus the traditional sigmoid function, is introduced to represent the relationship between lapses and economic factors. Theoretical features and practical applications of the new model are eventually discussed.

- Reverse mortgages through artificial intelligence: new opportunities for actuaries (Di Lorenzo, Piscopo, Sibillo, Tizzano). The reverse mortgage is a contract where a home owner borrows, partly or entirely, the future liquidation value of his home at the time of his death. The risks involved in the construction of the contract concern the house price (real estate risk), the interest rate (financial market risk) and the borrower's lifetime (longevity risk). The article explores the use of neural networks in order to obtain a predictive analysis of the pricing process. In the end, the authors suggest that their research can be the basis for future development in connecting financial with insurance products: e.g., the amount of the loan in their model could be linked to classic or sophisticated life insurance products, such as single premium life annuities.

- Modelling dynamic lapse with survival analysis and machine learning in CPI (Aleandri, Eletti). The article aims to describe, in a dynamic context, the behavior of policy-holders in credit protection insurance (CPI), i.e., in those products purchased by banks to cover the mortality risk of their borrowers. To this end, several machine learning models are compared in a survival analysis setting. Moreover, by simulating different interest rate scenarios, the combination of lapse estimation and insurance dynamics leads to a CPI profit model used to determine the time value of options and guarantees.

- Gaussian process regression for pricing variable annuities with stochastic volatility and interest rate (Goudenège, Molent, Zanette). The article investigates value and greeks of a guaranteed minimum withdrawal benefit variable annuity, when, in addition, an insurance product, a guaranteed minimum death benefit, is embedded in the contract. A numerical method solving the dynamic control problem, by computing optimal withdrawal, is employed. Precisely, a very fast machine learning technique, known as Gaussian Process Regression (GPR), is introduced. In fact, numerical experiments show that the accuracy of the values estimated by GPR is high with very low computational cost. Finally, the authors stress out that their method could be generalized to other insurance products.

- Challenges in approximating the Black and Scholes call formula with hyperbolic tangents (Mininni, Orlando, Taglialatela). The article's aim is to obtain a new approximating formula for the Black and Scholes call function through the hyperbolic tangent. Differently from other proposed solutions, this formula is invertible, which is particularly useful for extracting the implied volatility from quoted options. Hence, by comparing their results with the most common methods available in the literature, the authors discuss the challenges of their approach.

- Longevity risk and economic growth in sub-populations: evidence from Italy (Bozzo, Levantesi, Menzietti). Forecasting mortality is paramount for local and national definitions of economic policy. To this purpose, an ample literature exists about the long-run relationship between mortality evolution and a set of socioeconomic variables. Against such background, the article aims to investigate the link between mortality and real gross domestic product per capita (GDPPC) over 
time in Italian regions. In fact, a multi-population model is proposed including the level of real GDPPC. Such a model is compared to the classic Boonen-Li model and tested in out-of-sample forecasting experiment.

- Heterogeneity and uncertainty in a multi-state framework (Pitacco, Tabakova). The article develops a scheme based on a two-state model of one-year mortality, but the results turn out to hold also for other types of insurance events, such as disablement and accidents. In fact, the original scheme is extended into timediscrete models with more states (active- invalid-dead) in a multi-year time horizon. As a baseline probabilistic structure, a three-state model in a Markov context is adopted. The focus is on an insurance portfolio. The analysis of probability distributions allows to assess the risk profile of the portfolio, and thus to suggest appropriate action in terms of premium and capital allocations.

- On the determinants of data breaches: a co-integration analysis (De Giovanni, Leccadito, Pirra). The authors observe that cyber risks and particularly data breaches constitute an increasingly important frontier of risk modeling. In particular, they investigate short- and long-term relations between data breaches and bit-coinrelated variables. To this end, two different data sets of data breaches are assumed. In both cases, statistical evidence of a bidirectional lead-lag relation between the investigated variables in the short-run is provided. Moreover, the cointegration analysis performed by the authors suggests that this relation is likely to persist in the long-run. The quantitative implications of such relations are evaluated through a set of numerical techniques (Granger causality tests, impulse response analysis, variance decomposition of forecasting errors).

- Optimal annuitisation in a deterministic financial environment (Deelstra, Devolder, Melis). The reforms in public pension schemes have reduced post-retirement social security income. In order to compensate, individuals join pension funds and individual plans to increase their wealth at retirement. Post-retirement choices aimed to allocate the wealth at retirement between consumptions, risk free investment and a life annuity are analyzed in the article. Basically that leads to a discrete time optimization model, assuming a so-called constant relative risk aversion (CRRA) utility function. In particular, the effect of bequest motives and the optimal annuity rate used by the insurer is studied. Numerical applications illustrate optimal annuitisation results and optimal consumption paths.

- Flood risk resilience: the blockchain approach for an adaptive design of an insurance contract (Pagano, Romagnoli, Vannucci). The authors discuss how insurance markets can be used for mitigating the economic consequences of climate changes, in particular for facing flood risks. In fact, by an approach reminding the socalled resilience bonds, the authors propose financial instruments whose cash flows depend on the occurrence of contractually settled catastrophic events, whereas part of the investment is devoted to finance resilience actions. To this end, an adaptive design of the insurance contract is proposed, which has to be assessed by an engineering expertise, within a legal framework into which the actuarial quantitative model can be implemented. The periodic renewals of the contract can be interpreted as a sort of smart contracting, and in such framework a blockchain technology can be used to collect new information from a variety of sources.

In conclusion, we observe that, far from exhausting the growing variety and 
diversity of contemporary actuarial themes, the articles of this special issue can, however, provide a stimulating glimpse of the ever arising new problems in actuarial research and the multi-discipline (socio-economic, financial, mathematical, statistical, computer science) expertise required to deal with them.

Publisher's Note Springer Nature remains neutral with regard to jurisdictional claims in published maps and institutional affiliations. 